\title{
Mesoscale polycrystal calculations of damage in spallation in metals
}

\author{
D.L. Tonks, J. Bingert, V. Livescu, S. Luo, and C. Bronkhorst \\ Los Alamos National Laboratory, Los Alamos, NM 87545, USA
}

\begin{abstract}
The goal of this project is to produce a damage model forspallation in metals informed by the polycrystalline grain structure at themesoscale. Earlier damage models addressed the continuum macroscale in whichthese effects were averaged out. In this work we focus on cross sectionsfrom recovered samples examined with EBSD (electron backscattereddiffraction), which reveal crystal grain orientations and voids. We seek tounderstand the loading histories of specific sample regions by meshing upthe crystal grain structure of these regions and simulating the stress,strain, and damage histories in our hydrocode, FLAG. The stresses and strainhistories are the fundamental drivers of damage and must be calculated. Thecalculated final damage structures are compared with those from therecovered samples to validate the simulations.
\end{abstract}

The crystal plasticity model we use was drawn fromwork by Anand, Asaro, and Bronkhorst [1]. For illustration, Eq. (1) shows the basic plastic deformation rate tensor as a sum of plastic strain rates in the various crystal slip planes, labeled by $\alpha$ :

$$
L^{p}=\sum_{\alpha=1}^{n} \dot{\gamma}_{\alpha} \vec{s}^{\alpha} \vec{n}^{\alpha}
$$

The $\mathbf{s}$ and $\mathbf{n}$ are unit vectors tangential (in slipdirections) and normal to the crystal slip planes. The plastic strain rateon a given crystal plane, $\dot{\gamma}_{\alpha}$ was given by the following power law fitted to shock wave $\mathrm{Cu}$ polycrystal data [2]:

$$
\dot{\gamma}_{\alpha}=\left(\tau_{\alpha}-\tau_{o}-h \bar{\gamma}_{\alpha}\right)^{n}\left(\bar{\gamma}_{\alpha}+\bar{\gamma}_{o}\right)^{m}
$$

where $\tau_{\alpha}$ is the projected deviatoric stress and $\bar{\gamma}_{\alpha}$ is the accumulated plastic strain. The polycrystallinefit is not appropriate for single crystal simulations but should giveapproximate results.

The experiments simulated here involved columnar grain $\mathrm{Cu}$ samples with flyers thrown by the Trident laser at LANL. The simulations are 2D and are roughly appropriate for the columnar grains but are expected to miss theinfluence of material above and below the calculated plane. Figure 1 showsthe gas gun set up.

We simulated Shot 19071 which was of interest because the damage jumped upout of a defined spall plane to run a along a grain boundary (left arrow below). A simulation was done to find a possible explanation. Figure 2 shows the EBSD scan together with a simulated accumulated plastic strain field.

In this shot, the flyer velocity was $225 \mathrm{~m} / \mathrm{s}$ with a peak shot stress ofabout $4.3 \mathrm{GPa}$. The sample thickness was about $1 \mathrm{~mm}$. Note (arrows) that thereis a calculated plastic strain concentration along the lower grain boundary of the small "wedge" grain that coincides with the row of voids in the sample. In the simulation meshing, the flat, rectangular (orange) grain in the EBSD scan, to the left of the "wedge," was extended upward to the flyer boundary since only its lower grain boundary region had damage and was of interest. Each grain was meshed individually in this simulation, with the CUBIT software from SANDIA Labs.

In order to further explore the damage history of this shot, a macroscopicdamage model was added to the crystal plasticity modeling. This damage modelis a simplified version of the "Tonks Damage

This is an Open Access article distributed under the terms of the Creative Commons Attribution-Noncommercial License 3.0, which permits unrestricted use, distribution, and reproduction in any noncommercial medium, provided the original work is properly cited. 


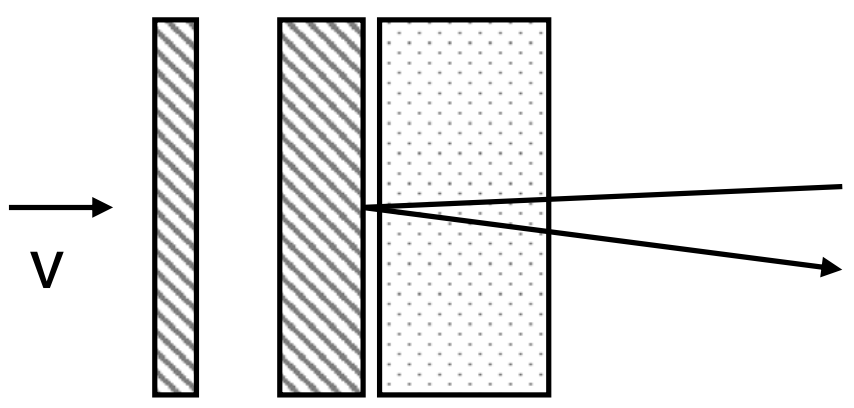

\section{Flyer Target Window}

Fig. 1. Typical gas gun flyer plate experimental configuration.
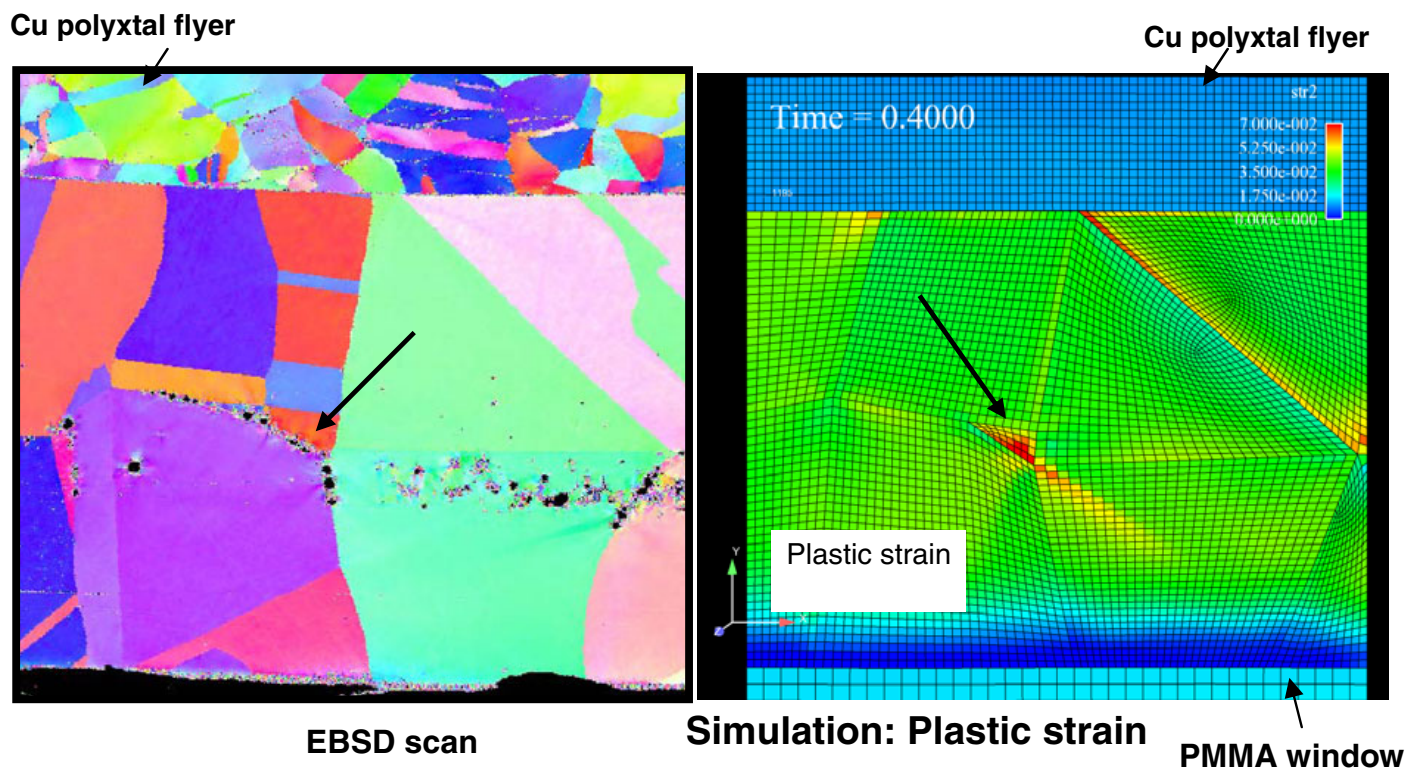

Fig. 2. EBSC scan (left) and calculated plastic strain (right) for shot 19071.

Model [3]." The modelcomponents of interest here include the nucleation model and the porositygrowth model. The hydrodynamic mesh variable is porosity, which is used todegrade or "soften" the elastic moduli. The nucleation is modeled as the instantaneous breakout due to negative pressure of small inclusions which have pressure stress breaking thresholds. The idea behind this model is debonding from inclusions.

The porosity growth model has an early, wave expansion phase, and a later phase with the stresses in equilibrium. In the wave growth phase, the voids expand rapidly to relieve the sudden stress imbalance of nucleation. For a typical pressure $\mathrm{P}$ at spallation, the void surface velocity is given by $\mathrm{V}$ in the formula: $\mathrm{P}=\mathrm{P}_{\mathrm{c}}+\alpha \mathrm{V}^{2}$, where $\alpha$ is approximately the mass velocity and $\mathrm{P}_{\mathrm{c}}$ is the cavitations pressure. This motion is inertia dominated.

After sufficient porosity growth, after stress waves have had opportunity to bring the stresses into equilibrium, a more conventional porosity growth law is used. This law belongs to the Gurson surface family. A pressure only version is used here for simplicity. This version is similar to, but not identical to, the Johnson spallation model [4]. The growth model is based on a spherical cell centered on a spherical void with pressure appliedat the outer boundary. The matrix material is viscoplastic with the 
New Models and Hydrocodes for Shock Wave Processes in Condensed Matter

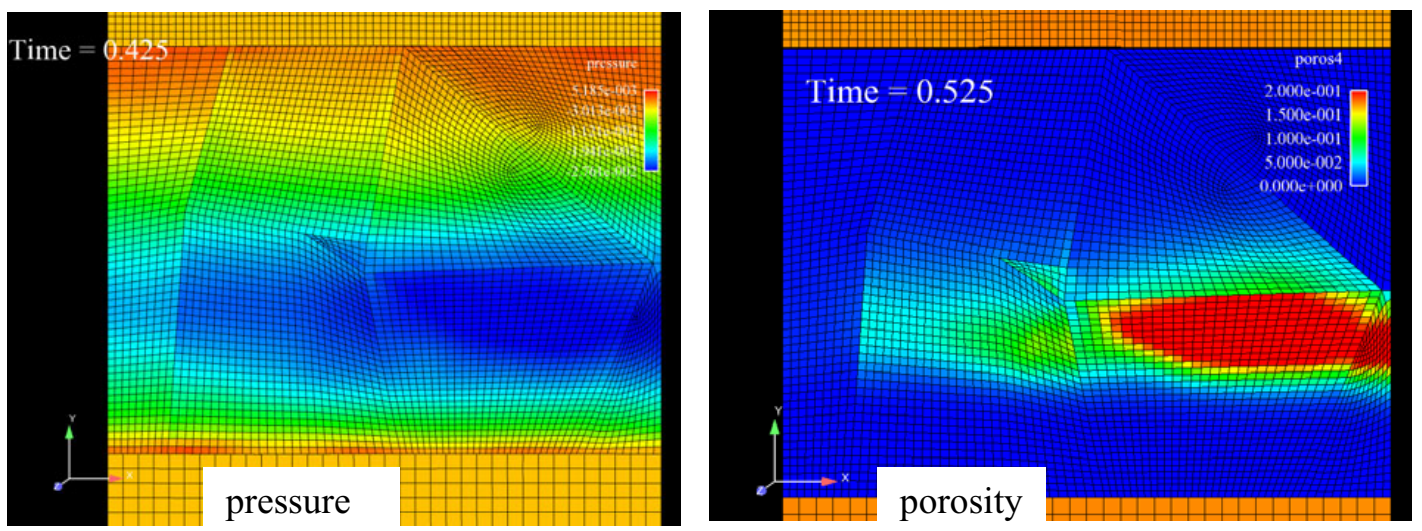

Fig. 3. Calculated pressure (left) and porosity (right) for Shot 19071.

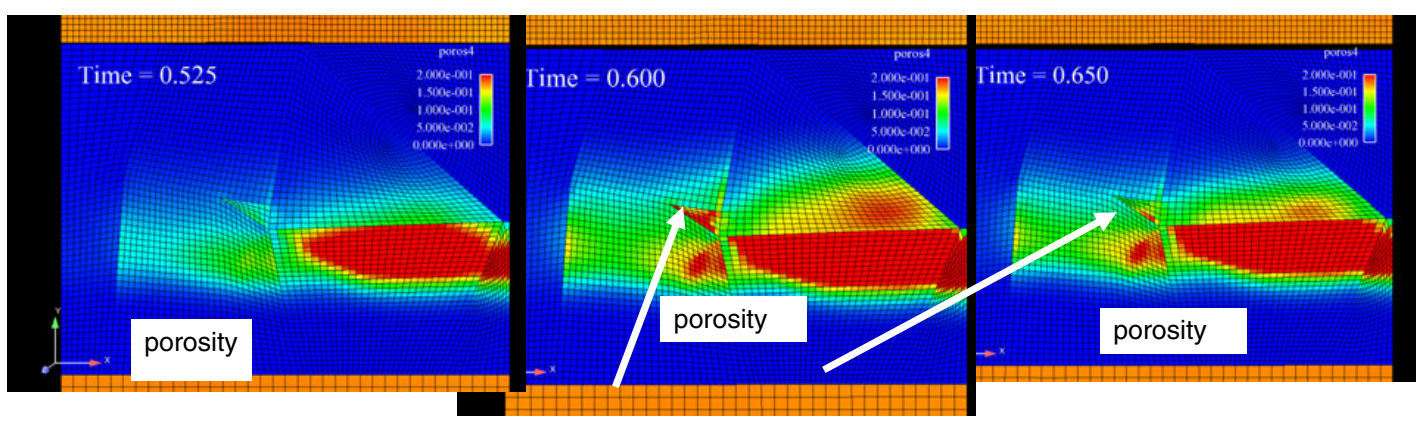

Fig. 4. Calculated porosity history for shot 19071.

plastic strain rate $\dot{\varepsilon}$ depending on the effective deviatoric stress, $\tau$, via the following power law Eq. (3), where y is a constant yield stress, which yields the porosity growth law Eq. (4):

$$
\begin{gathered}
\dot{\varepsilon}=\dot{\varepsilon}_{o}\left[(\tau-y) / \sigma_{o}\right]^{n} \\
\dot{\rho}=1.5 \dot{\varepsilon}_{o} \rho(1-\rho)\left[\left(-\frac{3}{2} P+y \ln \rho\right) /\left[\sigma_{o} n\left(1-\rho^{1 / n}\right)\right]\right]^{n}
\end{gathered}
$$

Note that the damage model responds only to negative pressure and not to anydeviatoric quantities. Thus, its simulated porosity response allows aseparation of pressure and deviatoric damage effects.

Figure 3, left panel, shows the pressure field at the first appearance ofsubstantial negative pressure. The pressures here range from $-2.76 \mathrm{GPa}$ to $0.52 \mathrm{GPa}$. The blue area is the negative pressure region where the release waves from the front of the flyer and the back of the sample have overlapped. At this time, $0.425 \mu$ s after impact, not much porosity has accumulated but, at a somewhat later time of $0.525 \mu \mathrm{s}$, the right panel shows significant porosity accumulation. Note the substantial porosity region to the right of the right panel which corresponds to negative pressure region.

Figure 4 below shows the porosity evolution past $0.525 \mu \mathrm{s}$, when porosity first develops in the small wedge grain and then crushes out somewhat (white arrows) due to repressurization. We should note that the porosity locations are of interest here, and not so much the magnitudes of damage, to draw conclusions about intergranular versus intragranular damage locations. The damage model was not adjusted to match the experimental levels of damage.

The pressure histories (not shown) indicate that the negative pressure hasmostly disappeared by $0.60 \mu$ s which explains why the porosity field does not change substantially after this time, except for the porosity crush out in the wedge grain, i.e. no new porosity is created after $0.60, \mu \mathrm{s}$. It should be 
noted that in order to bring out the porosity changes, the color contours have been chosen to saturate at 0.2 porosity (red) while the actual porosities range to 0.3 , the fracture point.

In comparing the EBSD scan in Figure 2 with the calculated porosity field above, note that the calculation predicts fairly well the intragranular porosity in the interior of the large trapezoidal (turquoise) grain just to the right of center, in the "spall plane."

However, the calculated porosity doesn't follow the grain boundary, or intergranular damage very well, except for the wedge grain which is small enough so that intergranular and intragranular damage have probably merged together. Since the damage model used here is driven by negative pressure, one can conclude that negative pressure can explain fairly well the damage interior to grains but not that at grain boundaries.

In earlier calculations for other shots, not shown here, in which no damagemodel was used, some correspondence was seen between damage and concentrations of deviatoric stress and strain at grain boundaries. Hence, it seems plausible that adding deviatoric stresses and strains to the damage model, in addition to the negative pressure already used, would permit a generalized damage model to capture damage in both locations. This avenue will be pursued in the future.

\section{References}

1. A. S. Khan and S. Huang, Continuum Theory of Plasticity (John Wiley, NY, 1995).

2. D. L. Tonks, "Rate-Dependent Plasticity of Copper and Stainless Steel Under Shock Compression," J. Applied Physics 66, 1951 (1989).

3. D. L. Tonks, W. R. Thissell, et al. (2003). "Modeling Incipient Copper Damage Data from the Tensile Hopkinson Bar and Gas Gun.” APS Topical ShockConference, AIP Conference Proceedings 706, Portland, OR, 507-510 (2003).

4. J. N. Johnson, "Dynamic Fracture and Spallation in Ductile Solids." J. Applied Physics 52, 28122825 (1981). 